\title{
The Relationship Between Different Types of Caries and Periodontal Disease Severity in Middle-Aged and Elderly People: Findings from the 4th National Oral Health Survey of China
}

\section{Lixia Yu (DYuu_LX@163.com)}

Guanghua School of Stomatology, Sun Yat-Sen University https://orcid.org/0000-0002-4133-4547

\section{Xing WANG}

Chinese Stomatological Association

\section{Xi Ping FENG}

Shanghai Jiao Tong University School of Medicine

\section{Bao Jun TAl}

School \& Hospital of Stomatology, Wuhan University

De Yu HU

West China Hospital of Stomatology Sichuan University

\section{Bo WANG}

Chinese Stomatological Association

\section{Chun Xiao WANG}

Chinese Center for Disease Control and Prevention

\section{Shu Guo ZHENG}

Peking University School and Hospital of Stomatology

Xue Nan LIU

Peking University School and Hospital of Stomatology

\section{Wen Sheng RONG}

Peking University School and Hospital of Stomatology

\section{Wei Jian WANG}

Peking University School and Hospital of Stomatology

\section{Yan SI}

Peking University School and Hospital of Stomatology

Huan Cai Lin

Hospital of Stomatology, Guanghua School of Stomatology, Sun Yat-sen University 
Keywords: dental caries, periodontal disease, epidemiology

Posted Date: June 25th, 2020

DOI: https://doi.org/10.21203/rs.3.rs-37354/v1

License: (c) (1) This work is licensed under a Creative Commons Attribution 4.0 International License. Read Full License 


\section{Abstract}

Background: To explore the relationship between different types of caries and periodontal disease severity in middle-aged and elderly people in China.

Methods: The study population consisted of 4,387 middle-aged and 3,225 elderly subjects. Caries was divided into three types: type A, crown caries; type B, lesions involving both the crown and root, which represents mixed type; and type $C$, root caries. These three types together represent the overall caries situation, which we call type ABC. Caries was quantitated by decayed and filled teeth (DFT index). Periodontitis was evaluated by clinical attachment loss.

Results: Middle-aged people with periodontitis had a significant association with DFT of type ABC (OR: 1.09, 95\% Cl: 1.06-1.11), type A (OR: 1.05, 95\% Cl: 1.02-1.08), type B (OR: 1.20, 95\% Cl: 1.16-1.28) and type $\mathrm{C}$ (OR: $1.36,95 \% \mathrm{Cl}: 1.15-1.60)$. Elderly people with periodontitis had a significant association with DFT of type ABC (OR: 1.05, 95\% Cl: 1.03-1.07), type B (OR: 1.07, 95\% Cl: 1.05-1.10) and type C (OR: 1.30, 95\% Cl: 1.19-1.42)『but not with type A.

Conclusions: Different types of caries in middle-aged people, the overall caries and caries types involving the root surface in elderly people were significantly associated with periodontal disease severity.

\section{Background}

Dental caries and periodontitis are the most common diseases in the oral cavity among adults. Both diseases are the primary cause of tooth loss and can lead to negative impacts upon quality of life. Over the years, the relationship between the two diseases is still controversial. Although several studies have shown a positive correlation between the two diseases [1-10], there are also some studies showing a negative correlation [11-13] or others showing no association [14].

The reasons for the positive correlation include common social-behavioural factors, such as age, gender, poor oral hygiene, smoking status, etc. [15]. In addition, there is evidence that host genetic factors play a moderate role in caries and periodontitis [15]. The main reason for the inverse correlation is different bacteriological spectrum. A great deal of evidence points to Streptococcus mutans as the primary cariogenic bacterium, while periodontitis is associated with specific gram-negative anaerobic bacteria, such as Porphyromonas gingivalis. A negative association has been reported between salivary levels of $S$. mutans and $P$. gingivalis, which was found in subjects with varying severity of caries and periodontitis, indicating that an inverse correlation exists between the two diseases [13].

Caries can be divided into three types according to the location of occurrence: crown caries, root caries and mixed type caries affecting both the crown and root. According to instructions from the WHO, if a carious lesion involves both the crown and the root, the possible site of origin of the carious lesion should be recorded as the site of decay. When it is impossible to determine the origin of the lesion, both the crown and root should be recorded as decayed [16]. In fact, in a large-scale oral epidemiological 
examination, it was very difficult to confirm the origin of caries in most cases. Therefore, it is difficult to classify the caries involving both the crown and root as coronal caries or root caries. As far as we know, in the study of the relationship between the two diseases, few studies have been able to clearly point out the specific types of caries they studied $[1,7]$. It has been pointed out that the detection methods of caries could be a plausible explanation for discrepancies found in the relationship between caries and periodontitis [12]. Due to the controversial relationship between the two diseases, it is necessary to classify the caries.

Inconsistencies in subject selection could be another plausible explanation for discrepancies found between the two diseases [12]. It has been pointed out that studies reporting an inverse association between the two diseases are mostly based on selected patients who were in a young population no more than 20 years of age having juvenile periodontitis, which was recognized as having increased susceptibility to periodontitis [14]. Like many diseases, age is an important factor that is greater than other known risk factors on caries and periodontitis to explain the variation in occurrence. The role of age in both diseases has been attributed to accumulated exposure. In addition, a recent study showed that susceptibility to the two diseases could change with ageing [17].

Taken together, the objective of this study is to explore the relationship between different types of caries and periodontal disease severity considering different types of caries in middle-aged and elderly people respectively from the 4th National Oral Health Survey in China. As caries and periodontitis are related to many social and behavioural background factors, ordered logistic regression was used for controlling relevant confounders.

\section{Methods}

\section{Sampling and clinical examining method}

The present study was a part of the 4th National Oral Health Survey, which is the most comprehensive oral health survey to date in China, carried out in 2015-2016. A multistage stratified sampling was used to recruit adults from all 31 provinces, municipalities and autonomous regions of mainland China. The probability proportional to size (PPS) method was used to randomly select subjects [18]. A total of 4,410 35- to 44-year-olds and 4,431 65- to 74-year-olds attended the survey. The exclusion criterion of this analysis was participants who had fewer than 20 teeth $[3,19]$ and periodontal status that cannot be examined for any reason, such as non-standard fixed prosthesis that cover the gingiva, or calculus is present to such an extent that periodontal examination is not possible.

The latest WHO recommendations (2013) were used for clinical oral examinations. The examinations were conducted by three trained licensed dentists, while three other trained individuals with clinical experience acted as recorders in each province. Examinations were conducted with a mobile dental chair, using artificial light, a disposable dental mirror, and a standard WHO Community Periodontal Index (CPI) probe [18]. 


\section{Variables}

\section{Independent variables}

Caries were recorded for all tooth surfaces, but observations were recorded by tooth. We divided caries into three types: type A, caries or a filling on the crown, which represents crown caries; type B, caries or a filling involving both the crown and root, which represents mixed type; and type $C$, caries or a filling on the root, which represents root caries. These three types together represent the overall caries situation, which we call type $A B C$ (Figure 1). Because residual roots involve both the crown and root, we classified residual roots as type $B$. The number of decayed and filled teeth (DFT) was calculated to analyse its relationship with periodontitis. The kappa values of the inter-examiners' reliabilities for the examination of dental caries were both 0.97 in the middle-aged and elderly group [18, 20].

\section{Dependent variables}

Periodontal disease severity was evaluated by clinical attachment loss (CAL) by tooth, including third molars. Probing was performed "walked around" along gingival crevices with a standardized force of no more than 20 grams. Each tooth was recorded according to severity. CAL was scored as 0 (0-3 mm), 1 (4 $-5 \mathrm{~mm}), 2$ (6-8 mm), 3 (9-11 mm), 4 (12 mm or more), 9 (tooth excluded) and X (tooth not present). Before the field investigation, examiners accepted training, and every examiner and a reference examiner carried out the examination on 10 to 15 subjects per group to assess the consistency. The kappa value was calculated and was more than 0.6 , which suggested good reliability [18].

\section{Covariates}

A face-to-face interview was conducted to collect information on participants' demographic characteristics: gender, area, education level, and household income per capita. Oral health behaviours: frequency of dessert consumption, frequency of tooth brushing, use of dental floss and toothpicks, smoking status, and alcohol consumption. Diabetes history [21, 22].

\section{Statistical analysis}

For analysis, periodontitis was divided into three groups according to CAL: $\leq 3 \mathrm{~mm}, 4-5 \mathrm{~mm}$, and $\geq 6$ $\mathrm{mm}$. Data analysis was carried out using SPSS 20.0. Categorical and continuous variables may have influenced caries status, and periodontitis was analysed using a Chi-square test and a Kruskal Wallis test, respectively. Those variables with $P<0.05$ were tested further in an ordered logistic regression to analyse the association between the two diseases with consideration of the other factors. Statistical analyses did not include missing values and the individual who preferred not to answer.

\section{Results}


A total of 4,387 subjects $35-44$ years old and 3,225 subjects $65-74$ years old were included in the analysis (Figure 2).

Table 1 shows the results of bivariate analysis of the characteristics of participants in relation to periodontal attachment loss in the 35 to 44 -year-old group. We found gender, area, education level, smoking status, tooth brushing frequency, use of dental floss, use of toothpicks, drinking alcohol, household income per capita and diabetes were significantly associated with periodontal disease severity $(P<0.05)$. To control for confounding factors, ordered logistic regression analyses were performed. Table 2 shows the association between the number of DFT and periodontal disease severity in the 35 to 44 year-old group. Adults with periodontitis had a significant association with DFT of type ABC (OR: 1.09, 95\% Cl: 1.06-1.11), type A (OR: 1.05, 95\% Cl: 1.02-1.08), type B (OR: 1.20, 95\% Cl: 1.16-1.28) and type C (OR: $1.36,95 \% \mathrm{Cl}: 1.15-1.60)$.

Table 3 shows the results of bivariate analysis of the characteristics of participants in relation to periodontal status in the 65 to 74 -year-old group. We found gender, area, smoking status, tooth brushing frequency, drinking alcohol and household income per capita were significantly associated with periodontal disease severity $(P<0.05)$. Table 4 shows the association between the number of DFT and periodontal disease severity in the 65 to 74 -year-old group. Elderly people with periodontitis had a significant association with DFT of type ABC (OR: 1.05, 95\% Cl: 1.03-1.07), type B (OR: 1.07, 95\% Cl: 1.051.10) and type $C$ (OR: $1.30,95 \%$ Cl: $1.19-1.42)$, but not with type A (OR: $0.98,95 \%$ Cl: 0.95-1.02, $P=0.331$ ).

\section{Discussion}

The commonly used criteria for defining periodontitis are probing depth (PD) and CAL. PD reflects the current situation of periodontitis, and CAL reflects the long-term accumulation of periodontal damage. Because caries and periodontitis are long-term chronic destructive diseases, the past cumulative lifetime experience of periodontitis is reflected by CAL measurements. Therefore, it is recommended to use this measure to analyse the risk factors of periodontitis in epidemiological studies [23].

DMFT, DT and DFT are the most commonly used indices to evaluate caries status and experience. In people aged 30 years or older, the M component of the DMFT index refers to the loss of teeth caused by any reason. To avoid the overestimation of the true extent of caries, we use the DFT index to analyse caries experience.

Previous studies on the relationship between caries and periodontitis have rarely clearly described whether the caries studied were crown caries, root caries, or mixed type. In recent years, there have been two articles clearly defining the types of caries studied-one was crown/root caries [1], and the other was crown caries [7]. Some studies have pointed out that, when we encounter type B caries, we do not know whether to classify it as crown caries or root caries. This is a measurement issue more than a diagnostic issue [24]. The relationship between caries and periodontitis in different locations for caries may be different, so we divided the types of caries in this way, hoping to further understand the relationship between caries and periodontitis. 
We found all four caries types $(A B C / A / B / C)$ were positively correlated with periodontitis in the middleaged group, and three caries types $(A B C / B / C)$ were positively correlated with periodontitis in the elderly group. In the past, few articles have discussed the relationship between the two diseases according to different age groups. There are studies reporting a positive correlation between caries and periodontal disease severity in adults. Al Qobaly et al. found individuals aged 35 years or older with periodontitis had a higher risk of coronal and root caries in England, Northern Ireland and Wales [1]. Hyman et al. reported that untreated coronal caries surfaces of US individuals aged 20 years or older was positively correlated with mean CAL [7]. Mattila et al. reported a positive association between PD and the DT index in Finnish adults who were 30 years and older [6]. Strauss et al. reported a positive association between periodontitis and the DT index of Chilean adults aged between 35 and 44 years [2]. The positive association could be explained by the common socioeconomic status they shared. Socioeconomic status seems to influence relevant health behaviours, such as diet, oral hygiene, smoking and the patterns of seeking professional prevention or treatment [15]. In our analysis, gender, area, smoking status, tooth brushing frequency and household income per capita showed a significant association with periodontitis in both age groups. As we know, both caries and periodontal diseases are bacterial infectious diseases. Although the microbiological profiles of the two are different, poor oral hygiene is considered one of the main common risk factors, which could provide enough fermentable carbohydrates for bacterial reproduction and subsequently increase the prevalence of the two diseases [1, 15]. In addition, smoking status has been proven to be another important health behaviour that is a common risk factor for both diseases [15]. Furthermore, it has been demonstrated that host genetics has a moderate role in contributing to the susceptibility of both caries and periodontal disease. Although no common genetic genes were found, pleiotropy (one gene influences two or more seemingly unrelated phenotypic traits) in caries and periodontal disease may exist. Hence, with regards to the role of genetics, more analysis of pleiotropy is needed to unravel the mechanism to better understand the genetic association of caries and periodontal disease accumulating in the same person [15].

Type A caries were shown to have no relationship, but types B and C were positively associated with periodontitis in the elderly group in our study. In the middle-aged group, the DFT index increased with the aggravation of periodontitis, while in the elderly, the group with the most severe CAL ( $\geq 6 \mathrm{~mm}$ ) had the lowest DFT. Tooth loss may be a cause. With the increase of age, the tooth loss rate of the elderly increased significantly, and the most frequent occurrence of tooth extraction due to periodontitis should be in the group with $C A L \geq 6 \mathrm{~mm}$. But because of the cross-sectional study, we could not investigate the specific reasons for tooth extraction. Carious lesions of both types $B$ and $C$ involve the root surface. Periodontal attachment loss could lead to exposure of the root surface. As a result of poor oral hygiene, root caries commonly presents as a progressive lesion in patients with periodontitis [25]. The positive correlation between types $\mathrm{B}$ and $\mathrm{C}$ caries and periodontitis is consistent with a recent systemic review. Root caries incidence or increment has been reported to be associated with patients having periodontal disease [17].

This is the first study to analyse the relationship between caries and periodontal disease in a representative national sample of Chinese adults. We discuss the relationship between the two diseases 
by age group, because the risk factors, susceptibility of both diseases, and especially the number and causes of missing teeth in different age groups are different. However, there are some potential limitations in this study that should be addressed. First, due to the cross-sectional data of this study, the causal relationship between caries and periodontitis cannot be concluded. Second, we were obligated to identify periodontitis using CAL instead of definitions of periodontitis, such as that described by the Centers for Disease Control and Prevention and the American Academy of Periodontology (CDC-AAP).

\section{Conclusions}

A positive association between different caries types and periodontal disease severity was found in middle-aged people in China. Except crown caries, the overall caries and caries types involving the root surface were positively associated with periodontal disease severity in elderly people.

\section{Abbreviations}

CAL: Clinical attachment level; CDC-AAP: Center for Disease Control and Prevention and the American Academy of Periodontology; CPI: Community Periodontal Index; DFT: Decayed and filled teeth; PD: Probing depth; PPS: Probability proportional to size; WHO: World Health Organization

\section{Declarations}

\section{Ethics approval and consent to participate}

This study was approved by the Stomatological Ethics Committee of the Chinese Stomatological Association (Approval no. 2014-003). Informed written consent was obtained from each participant.

\section{Consent for publication}

Not applicable.

\section{Availability of data and materials}

The datasets analysed in this study are available from the corresponding authors on reasonable request.

\section{Competing interests}

The authors have stated explicitly that there are no conflicts of interest in connection with this article.

\section{Funding}


This study was supported by the Scientific Research Fund of National Health Commission of the People's Republic of China (201502002).

\section{Authors' contributions}

Dr Li Xia YU contributed to the data analysis and preparation of the manuscript; Drs Xing WANG, Xi Ping FENG, Bao Jun TAl, De Yu HU, Bo WANG, Chun Xiao WANG, Shu Guo ZHENG, Xue Nan LIU, Wen Sheng RONG, Wei Jian WANG, Yan SI, and Huan Cai LIN trained the investigators, designed and supervised the survey; Drs Yan SI and Huan Cai LIN contributed to the design of the study, general supervision of the research group, and critically revised the manuscript for important intellectual content.

\section{Acknowledgements}

Not applicable.

\section{References}

1. AlQobaly L, Sabbah W. The association between periodontal disease and root/coronal caries. Int J Dent Hyg. 2019;18(1):99-106.

2. Strauss F, Espinoza I, Stähli A, Baeza M, Cortés R, Morales A, Gamonal J. Dental caries is associated with severe periodontitis in Chilean adults: a cross-sectional study. BMC oral health. 2019;19(278).

3. Durand R, Roufegarinejad A, Chandad F, Rompré PH, Voyer R, Michalowicz BS, Emami E. Dental caries are positively associated with periodontal disease severity. Clin Oral Investig. 2019;23(10):3811-9.

4. Nascimento GG, Baelum V, Dahlen G, Lopez R. Methodological issues in assessing the association between periodontitis and caries among adolescents. Community Dent Oral Epidemiol. 2018;46(3):303-9.

5. Zimmermann H, Hagenfeld D, Diercke K, El-Sayed N, Fricke J, Greiser KH, et al. Pocket depth and bleeding on probing and their associations with dental, lifestyle, socioeconomic and blood variables: a cross-sectional, multicenter feasibility study of the German National Cohort. BMC oral health. 2015;15(7).

6. Mattila PT, Niskanen MC, Vehkalahti MM, Nordblad A, Knuuttila ML. Prevalence and simultaneous occurrence of periodontitis and dental caries. J Clin Periodontol. 2010;37(11):962-7.

7. Hyman JJ, Reid BC. Epidemiologic risk factors for periodontal attachment loss among adults in the United States. J Clin Periodontol. 2003;30(3):230-7.

8. Elter JR, Beck JD, Slade GD, Offenbacher S. Etiologic models for incident periodontal attachment loss in older adults. J Clin Periodontol. 1999;26(2):113-23.

9. Albandar JM, Brown LJ, Löe H. Dental caries and tooth loss in adolescents with early-onset periodontitis. J Periodontol. 1996;67(10):960-7. 
10. Albandar JM, Buischi YA, Axelsson P. Caries lesions and dental restorations as predisposing factors in the progression of periodontal diseases in adolescents. A 3-year longitudinal study. J Periodontol. 1995;66(4):249-54.

11. Al-Habashneh R, Al-Omari MA, Taani DQ. Smoking and caries experience in subjects with various form of periodontal diseases from a teaching hospital clinic. Int J Dent Hyg. 2009;7(1):55-61.

12. Sioson PB, Furgang D, Steinberg LM, Fine DH. Proximal caries in juvenile periodontitis patients. J Periodontol. 2000;71(5):710-6.

13. Iwano Y, Sugano N, Matsumoto K, Nishihara R, lizuka T, Yoshinuma N, Ito K. Salivary microbial levels in relation to periodontal status and caries development. J Periodontal Res. 2010;45(2):165-9.

14. Kinane DF, Jenkins WM, Adonogianaki E, Murray GD: Cross-sectional assessment of caries and periodontitis risk within the same subject. Community Dent Oral Epidemiol. 1991;19(2):78-81.

15. Chapple ILC, Bouchard P, Cagetti MG, Campus G, Carra M, Cocco F, et al. Interaction of lifestyle, behaviour or systemic diseases with dental caries and periodontal diseases: consensus report of group 2 of the joint EFP/ORCA workshop on the boundaries between caries and periodontal diseases. J Clin Periodontol. 2017;44(Suppl 18):S39-S51.

16. WHO. Oral health surveys: Basic methods. 5th ed. Geneva: World Health Organization; 2013.

17. López R, Smith PC, Göstemeyer G, Schwendicke F. Ageing, dental caries and periodontal diseases. J Clin Periodontol. 2017;44(Suppl 18):S145-S52.

18. Lu HX, Tao DY, Lo ECM, Li R, Wang X, Tai BJ, et al. The 4th National Oral Health Survey in the Mainland of China: Background and Methodology. Chin J Dent Res. 2018;21(3):161-5.

19. Guo J, Ban JH, Li G, Wang X, Feng XP, Tai BJ, et al. Status of Tooth Loss and Denture Restoration in Chinese Adult Population: Findings from the 4th National Oral Health Survey. Chin J Dent Res. 2018;21(4):249-57.

20. Gao YB, Hu T, Zhou XD, Shao R, Cheng R, Wang GS, et al. Dental Caries in Chinese Elderly People: Findings from the 4th National Oral Health Survey. Chin J Dent Res. 2018;21(3):213-20.

21. Sun HY, Jiang H, Du MQ, Wang X, Feng XP, Hu DY, et al. The Prevalence and Associated Factors of Periodontal Disease among 35 to 44-year-old Chinese Adults in the 4th National Oral Health Survey. Chin J Dent Res. 2018;21(4):241-7.

22. Wang CX, Ma LL, Yang Y, Xu MR, Wang X, Feng XP, et al. Oral Health Knowledge, Attitudes, Behaviour and Oral Health Status of Chinese Diabetic Patients Aged 55 to 74 Years. Chin J Dent Res. 2018;21(4):267-73.

23. Tonetti MS, Claffey N. Advances in the progression of periodontitis and proposal of definitions of a periodontitis case and disease progression for use in risk factor research - Group C Consensus report of the 5th European workshop in periodontology. J Clin Periodontol. 2005;32(Suppl 6):S210-S3.

24. Banting DW. The diagnosis of root caries. J Dent Educ. 2001; 65(10):991-6.

25. Bignozzi I, Crea A, Capri D, Littarru C, Lajolo C, Tatakis DN. Root caries: a periodontal perspective. J Periodontal Res. 2014;49(2):143-63. 


\section{Tables}

Table 1 Bivariate analysis of participant characteristics in relation to periodontal status in the middleaged group. 


\begin{tabular}{|c|c|c|c|c|}
\hline Variables & $\begin{array}{l}C A L \leq 3 \mathrm{~mm} \\
\mathrm{~N}=2938\end{array}$ & $\begin{array}{l}C A L=4-5 \mathrm{~mm} \\
\mathrm{~N}=1118\end{array}$ & $\begin{array}{l}\mathrm{CAL} \geq 6 \mathrm{~mm} \\
\mathrm{~N}=331\end{array}$ & $P$ value $^{a}$ \\
\hline Gender (\%) & & & & $<0.001$ \\
\hline Female & 54.4 & 44.8 & 30.8 & \\
\hline Male & 45.6 & 55.2 & 69.2 & \\
\hline Area (\%) & & & & $<0.001$ \\
\hline Urban & 52.6 & 45.4 & 52.6 & \\
\hline Rural & 47.4 & 54.6 & 47.4 & \\
\hline Educational level (\%) & & & & $<0.001$ \\
\hline$\leq 12$ years & 69.0 & 78.4 & 81.0 & \\
\hline$>12$ years & 31.0 & 21.6 & 19.0 & \\
\hline Smoking status (\%) & & & & $<0.001$ \\
\hline Never & 70.8 & 61.2 & 53.2 & \\
\hline Current (former) & 29.2 & 38.8 & 46.8 & \\
\hline Tooth brushing frequency (\%) & & & & $<0.001$ \\
\hline$<$ Once per day & 4.6 & 10.1 & 13.3 & \\
\hline$\geq$ Once per day & 95.4 & 89.9 & 86.7 & \\
\hline Use of dental floss (\%) & & & & $<0.001$ \\
\hline No & 93.8 & 96.8 & 96.7 & \\
\hline Yes & 6.2 & 3.2 & 3.3 & \\
\hline Use of toothpick (\%) & & & & $<0.001$ \\
\hline No & 57.0 & 50.4 & 43.5 & \\
\hline Yes & 43.0 & 49.6 & 56.5 & \\
\hline Drinking alcohol (\%) & & & & 0.019 \\
\hline Ceased & 2.3 & 2.9 & 4.5 & \\
\hline Scarcely/never & 85.4 & 82.5 & 78.9 & \\
\hline Daily & 4.4 & 5.2 & 5.4 & \\
\hline Weekly & 7.8 & 9.5 & 11.2 & \\
\hline Missing values ${ }^{b}$ & 0.1 & 0.0 & 0.0 & \\
\hline
\end{tabular}




\begin{tabular}{|lllll|}
\hline Frequency of dessert consumption (\%) & \multicolumn{5}{l|}{0.156} \\
\hline < Twice a day & 97.5 & 98.5 & 97.9 & \\
\hline$\geq$ Twice a day & 2.5 & 1.5 & 2.1 & $<0.001$ \\
\hline Household income per capita (\%) & & & & \\
\hline Less than RMB5,000/person & 18.7 & 24.8 & 28.4 & \\
\hline RMB5,000 to RMB15,000/person & 32.2 & 30.5 & 32.9 & \\
\hline More than RMB15,000/person & 33.4 & 28.4 & 26.6 & \\
\hline Prefer not to answer ${ }^{\text {c }}$ & 15.7 & 16.3 & 12.1 & \\
\hline Diabetes (\%) & & & & \\
\hline No & 98.5 & 97.9 & 94.9 & \\
\hline Yes & 1.5 & 2.1 & 5.1 & \\
\hline
\end{tabular}

${ }^{\text {a }}$ Chi-square test.

Covariates with $P<0.05$ were included in the ordered logistic regression.

$b, c$ Statistical analyses did not include missing values and the individual who preferred not to answer.

Table 2 The association between dental caries and periodontal disease severity in the middle-aged group.

\begin{tabular}{|c|c|c|c|c|c|}
\hline $\begin{array}{l}\text { DFT } \\
(\text { mean } \pm S D)\end{array}$ & $\begin{array}{l}C A L \leq 3 \mathrm{~mm} \\
\mathrm{~N}=2938\end{array}$ & $\begin{array}{l}C A L=4-5 \mathrm{~mm} \\
\mathrm{~N}=1118\end{array}$ & $\begin{array}{l}\mathrm{CAL} \geq 6 \mathrm{~mm} \\
\mathrm{~N}=331\end{array}$ & $P$ value $^{\mathrm{a}}$ & $\begin{array}{l}\text { Adjusted } P \text { value and } \\
\text { OR }(95 \% \mathrm{Cl})^{b}\end{array}$ \\
\hline Type ABC & $1.92 \pm 2.69$ & $2.40 \pm 3.08$ & $2.91 \pm 3.42$ & $<0.001$ & $\begin{array}{l}<0.001 \\
1.09(1.06,1.11)\end{array}$ \\
\hline Type A & $1.52 \pm 2.22$ & $1.66 \pm 2.32$ & $1.67 \pm 2.18$ & 0.024 & $\begin{array}{l}0.001 \\
1.05(1.02,1.08)\end{array}$ \\
\hline Type B & $0.37 \pm 1.13$ & $0.69 \pm 1.55$ & $1.11 \pm 2.10$ & $<0.001$ & $\begin{array}{l}<0.001 \\
1.20(1.16,1.28)\end{array}$ \\
\hline Type C & $0.03 \pm 0.28$ & $0.05 \pm 0.41$ & $0.13 \pm 0.62$ & $<0.001$ & $\begin{array}{l}<0.001 \\
1.36(1.15,1.60)\end{array}$ \\
\hline
\end{tabular}


${ }^{\text {a }}$ Kruskal Wallis test.

b Ordered logistic regression adjusted for gender, area, education level, smoking status, tooth brushing frequency, use of dental floss, use of toothpick, drinking alcohol, household income per capita and diabetes.

Table 3 Bivariate analysis of participant characteristics in relation to periodontal status in the elderly group. 
Variables

\begin{tabular}{|c|c|c|c|c|}
\hline & $N=728$ & $\mathrm{~N}=1202$ & $\mathrm{~N}=1295$ & \\
\hline Gender (\%) & & & & $<0.001$ \\
\hline Female & 58.7 & 53.4 & 42.2 & \\
\hline Male & 41.3 & 46.6 & 57.8 & \\
\hline Area (\%) & & & & 0.040 \\
\hline Urban & 56.7 & 50.9 & 52.1 & \\
\hline Rural & 43.3 & 49.1 & 47.9 & \\
\hline Educational level (\%) & & & & 0.492 \\
\hline$\leq 12$ years & 92.4 & 93.3 & 93.9 & \\
\hline$>12$ years & 7.6 & 6.7 & 6.1 & \\
\hline Smoking status (\%) & & & & $<0.001$ \\
\hline Never & 70.1 & 68.1 & 55.8 & \\
\hline Current (former) & 29.9 & 31.9 & 44.2 & \\
\hline Tooth brushing frequency (\%) & & & & $<0.001$ \\
\hline$<$ Once per day & 11.1 & 15.3 & 17.8 & \\
\hline$\geq$ Once per day & 88.9 & 84.7 & 82.2 & \\
\hline Use of dental floss (\%) & & & & 0.365 \\
\hline No & 97.5 & 97.6 & 98.3 & \\
\hline Yes & 2.5 & 2.4 & 1.7 & \\
\hline Use of toothpick (\%) & & & & 0.131 \\
\hline No & 46.8 & 42.2 & 44.4 & \\
\hline Yes & 53.2 & 57.8 & 55.6 & \\
\hline Drinking alcohol (\%) & & & & 0.030 \\
\hline Ceased & 7.3 & 8.8 & 9.3 & \\
\hline Scarcely/never & 80.9 & 79.5 & 74.9 & \\
\hline Daily & 8.2 & 8.2 & 10.9 & \\
\hline Weekly & 3.6 & 3.6 & 4.8 & \\
\hline Missing values ${ }^{b}$ & 0.0 & 0.0 & 0.1 & \\
\hline
\end{tabular}

Page 15/18 
Frequency of dessert consumption (\%)

0.237

\begin{tabular}{lllll}
\hline$<$ Twice a day & 98.5 & 98.8 & 98.0 & \\
\hline$\geq$ Twice a day & 1.5 & 1.2 & 2.0 & 0.040 \\
\hline Household income per capita (\%) & & & & \\
\hline Less than RMB5,000/person & 24.2 & 29.9 & 29.9 & \\
\hline RMB5,000 to RMB15,000/person & 26.9 & 23.3 & 24.4 & \\
\hline More than RMB15,000/person & 29.7 & 27.8 & 27.2 & \\
\hline Prefer not to answer ${ }^{c}$ & 19.2 & 19.0 & 18.5 & \\
\hline Diabetes (\%) & & & & \\
\hline No & 87.1 & 87.3 & 87.6 & \\
\hline Yes & 12.9 & 12.7 & 12.4 &
\end{tabular}

${ }^{a}$ Chi-square test.

Covariates with $P<0.05$ were included in the ordered logistic regression.

b,c Statistical analyses did not include missing values and the individual who preferred not to answer.

Table 4 The association between dental caries and periodontal disease severity in the elderly group.

\begin{tabular}{|c|c|c|c|c|c|}
\hline $\begin{array}{l}\text { DFT } \\
(\text { mean } \pm S D)\end{array}$ & $\begin{array}{l}\mathrm{CAL} \leq 3 \mathrm{~mm} \\
\mathrm{~N}=728\end{array}$ & $\begin{array}{l}C A L=4-5 \mathrm{~mm} \\
\mathrm{~N}=1202\end{array}$ & $\begin{array}{l}C A L \geq 6 \mathrm{~mm} \\
\mathrm{~N}=1295\end{array}$ & $P$ value $^{\text {a }}$ & $\begin{array}{l}\text { Adjusted } P \text { value and } \\
\text { OR }(95 \% \mathrm{Cl})^{b}\end{array}$ \\
\hline \multirow[t]{2}{*}{ Type ABC } & $3.35 \pm 4.26$ & $4.00 \pm 4.28$ & $4.30 \pm 4.49$ & $<0.001$ & $<0.001$ \\
\hline & & & & & $1.05(1.03,1.07)$ \\
\hline \multirow[t]{2}{*}{ Type A } & $1.46 \pm 2.19$ & $1.53 \pm 2.10$ & $1.24 \pm 1.80$ & 0.002 & 0.331 \\
\hline & & & & & $0.98(0.95,1.02)$ \\
\hline \multirow[t]{2}{*}{ Type B } & $1.76 \pm 3.35$ & $2.18 \pm 3.25$ & $2.65 \pm 1.15$ & $<0.001$ & $<0.001$ \\
\hline & & & & & $1.07(1.05,1.10)$ \\
\hline \multirow[t]{2}{*}{ Type C } & $0.13 \pm 0.57$ & $0.29 \pm 1.05$ & $0.41 \pm 1.15$ & $<0.001$ & $<0.001$ \\
\hline & & & & & $1.30(1.19,1.42)$ \\
\hline
\end{tabular}

${ }^{\text {a }}$ Kruskal Wallis test. 
b Ordered logistic regression adjusted for gender, area, smoking status, tooth brushing frequency, drinking alcohol and household income per capita.

Figures

\begin{tabular}{|c|}
\hline \multicolumn{2}{|c|}{ Total sample size of individuals } \\
Middle-aged group:4410 $\quad$ Elderly group: 4431 \\
\hline
\end{tabular}

Exclusion of : Participants who had fewer than 20 teeth

Middle-aged group: $4388 \quad$ Elderly group:3259

Exclusion of : Participants who's periodontal status cannot be examined

Middle-aged group: $4387 \quad$ Elderly group: 3225

Were included in the final analysis

Figure 1

Flow chart of data collection.

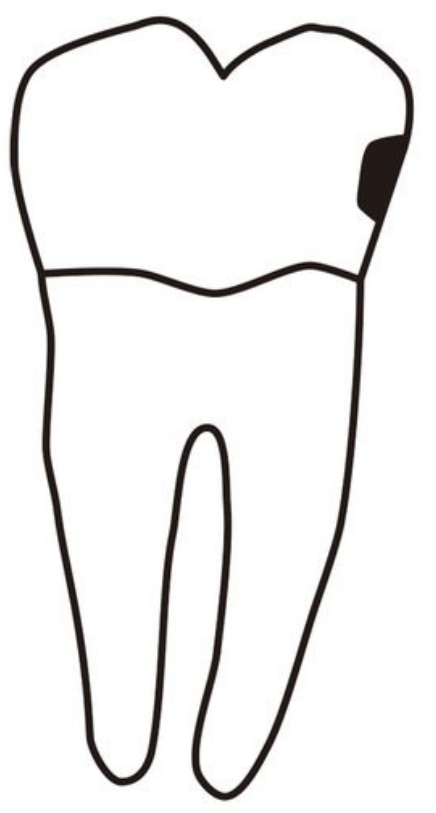

A

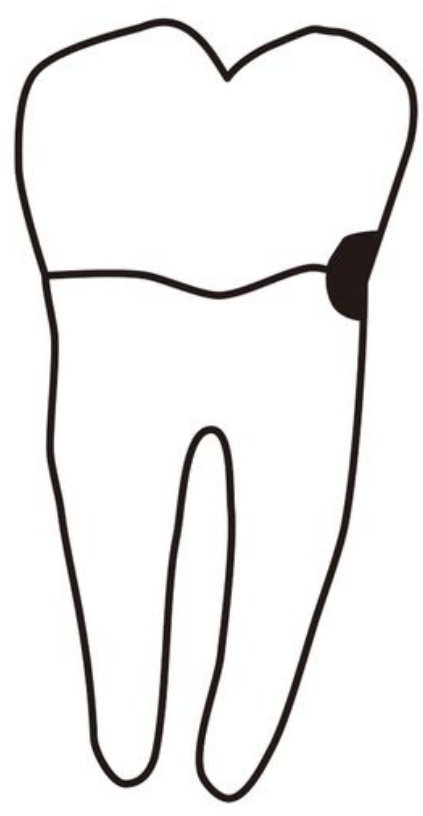

B

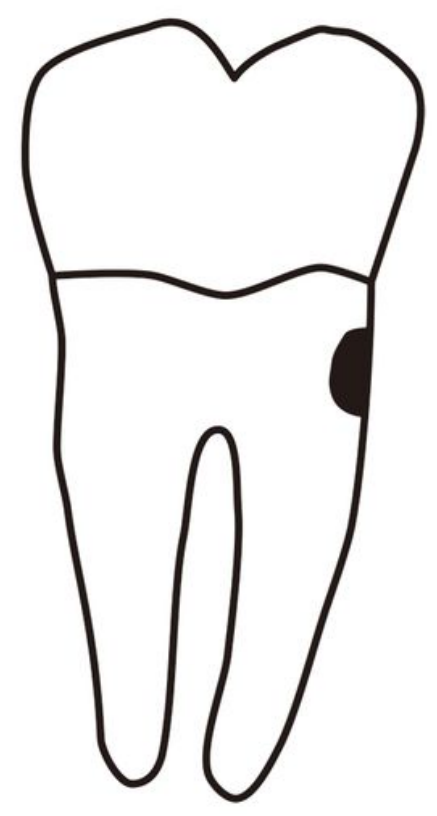

C

Figure 2 
Types of dental caries: which are divided according to the occurrence location. Legends: Type A, caries or a filling on the crown which represents crown caries. Type B, caries or a filling involve both the crown and root which represents mixed type. Type $\mathrm{C}$, caries or a filling on the root which represents root caries. These three types together represent the overall caries situation, which we call type ABC. 